\title{
Mass Efficiency Considerations for Thermally Insulated Structural Skin of an Aerospace Vehicle
}

\author{
Max L. Blosser* \\ NASA Langley Research Center, 23681, USA
}

\begin{abstract}
An approximate equation was derived to predict the mass of insulation required to limit the maximum temperature reached by an insulated structure subjected to a transient heating pulse. In the course of the derivation two figures of merit were identified. One figure of merit correlates to the effectiveness of the heat capacity of the underlying structural material in reducing the amount of required insulation. The second figure of merit provides an indicator of the mass efficiency of the insulator material. An iterative, one dimensional finite element analysis was used to size the external insulation required to protect the structure at a single location on the Space Shuttle Orbiter and a reusable launch vehicle. Required insulation masses were calculated for a range of different materials for both structure and insulator. The required insulation masses calculated using the approximate equation were shown to typically agree with finite element results within 10 to 20 percent over the range of parameters studied. Finite element results closely followed the trends indicated by both figures of merit.
\end{abstract}

\section{Nomenclature}

$c_{p e} \quad$ Effective insulator specific heat capacity

$c_{p s} \quad$ Structural specific heat capacity

$d_{e} \quad$ Insulator thickness

$d_{s} \quad$ Structural thickness

$k_{e} \quad$ Effective insulator thermal conductivity

$m \quad$ Mass per unit area

$m_{e} \quad$ Insulation mass per unit area

$m_{s} \quad$ Structural mass per unit area

$m_{\text {opt }} \quad$ Minimum total mass

$m_{\text {so }} \quad$ Structural mass at which total mass is minimum

$P_{a v g}$ Average ambient pressure during equivalent square surface temperature pulse

$T \quad$ Temperature

$t \quad$ Time

$T_{h} \quad$ Applied surface temperature rise of insulator

$t_{h} \quad$ Duration of heating pulse

$T_{i} \quad$ Initial temperature

$T_{m} \quad$ Maximum structural temperature rise

$T_{c e} \quad$ Temperature to use for calculating effective insulation specific heat capacity

$T_{c s} \quad$ Temperature to use for calculating effective structural specific heat capacity

$T_{k e} \quad$ Temperature to use for calculating effective insulation conductivity

\section{Symbols}

$\beta_{s} \quad$ Figure of merit for thermal performance of structure

$\gamma \quad$ Ratio of insulator to structural heat capacitance

$\kappa_{e} \quad$ Figure of merit for thermal performance of insulator

${ }^{*}$ Research Engineer, Structural Mechanics and Concepts Branch 
$\rho_{e} \quad$ Effective insulator density

$\rho_{s} \quad$ Structural density

$\tau_{h} \quad$ Non-dimensionalized duration of heat pulse

\section{Introduction}

AROSPACE vehicles in hypersonic flight generally require some form of thermal protection system to Aaccommodate the aerodynamic heating on the vehicle surface. One approach is to cover the exterior surface with a thermal insulator, such as the reusable ceramic tiles and blankets used on the Space Shuttle Orbiter. Typically, the vehicle structure would be designed for structural considerations. The insulation would then be sized to maintain the structure below the maximum allowable temperature for all design environments and loads. However, the amount of required insulation is a function of the mass and properties of the underlying structure. Also, it is not clear what combination of insulation properties would lead to the lightest insulation for the transient thermal surface heating.

In this paper an approximate solution ${ }^{1,2}$ for maximum structural temperature of an insulated structure is used to explore parameters that drive the combined mass of structure and insulator. Equations are developed for the minimum insulator mass required to maintain the inner, unheated surface below a specified temperature. A figure of merit which correlates to the effectiveness of the heat capacity of the underlying structural material in reducing the amount of required insulation was developed. A second figure of merit was identified for the combination of insulator thermal properties that minimizes the mass of an insulator required to limit the maximum temperature of an underlying structure subjected to a transient heating pulse. Insulation was sized for a number of surface heating histories, insulators, and structural materials by iterating a one-dimensional, finite element analysis and by using the simple equations derived in this paper. Finite element results were correlated to the two figures of merit developed in this paper.

\section{Problem Definition}

The simplified thermal problem that was analyzed by Blosser ${ }^{1,2}$ is illustrated in Figure 1. A thermal insulator with thickness, $d_{e}$, density, $\rho_{e}$, specific heat capacity, $c_{p e}$, and thermal conductivity, $k_{e}$ covers a structure (inner face sheet) of thickness, $d_{s}$, density, $\rho_{s}$, and specific heat capacity, $c_{p s}$. The inner surface of the structure is assumed to be perfectly insulated to simplify the mathematics of the problem and because it is a commonly used conservative assumption for sizing thermal protection systems. To further simplify the problem, the structure is treated as a lumped heat capacitance. For this solution the material properties are assumed to be constant, so

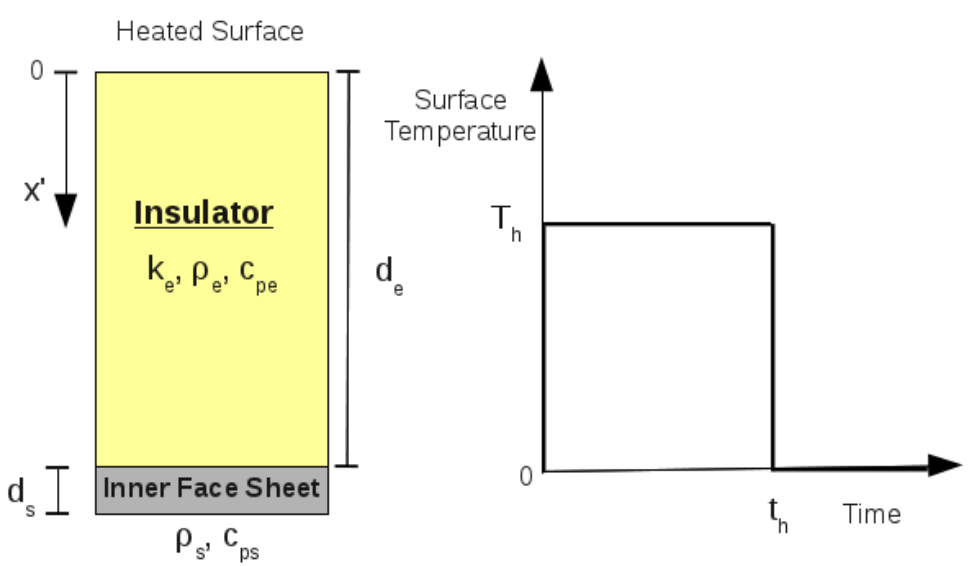

Figure 1. Illustration of simplified problem effective, averaged properties would have to be used to approximate more complex material behavior.

A simple transient heat pulse was assumed. Initially the insulator and structure are assumed to be at a uniform temperature of 0 . The outer surface of the insulator is assumed to instantaneously rise to a temperature $T_{h}$ at $t=0$ and maintain that temperature until $t=t_{h}$ at which time it instantaneously returns to 0 .

\section{Maximum Structural Temperature}

An approximate solution ${ }^{2}$ developed for the maximum structural temperature of the simplified problem illustrated in Figure 1 is given by: 


$$
\left(\frac{T_{m}}{T_{h}}\right)_{a}=1-e^{-\tau_{h} \sqrt{\frac{\gamma}{2}}}
$$

where

$$
\gamma=\frac{\rho_{e} c_{p e} d_{e}}{\rho_{s} c_{p s} d_{s}}
$$

and

$$
\tau_{h}=\frac{k_{e}}{\rho_{e} c_{p e} d_{e}^{2}} t_{h}
$$

\section{Mass of Insulated Structure}

For aerospace vehicles reducing mass is of utmost importance. Therefore, it is helpful to develop further insight into the interplay between the mass of structure and the mass of insulator required to limit the structural temperature. The mass per unit area of this simplified, insulated structure can be written simply as:

$$
m=m_{e}+m_{s}=\rho_{e} d_{e}+\rho_{s} d_{s}
$$

The relationship between $d_{e}$ and $d_{s}$ that is required to limit the maximum structural temperature to a specified value is given by Equation (1). By substituting Equations (2) and (3) into Equation (1) and rearranging terms, the following expression can be obtained:

$$
d_{s} d_{e}^{3}=\frac{\left(k_{e} t_{h}\right)^{2}}{2\left(\rho_{s} c_{p s}\right)\left(\rho_{e} c_{p e}\right)\left(-\ln \left(1-\frac{T_{m}}{T_{h}}\right)\right)^{2}}
$$

The equation can be further manipulated to give an explicit expression for $d_{e}$. This provides a very useful equation to calculate the insulator thickness required to limit the maximum structural temperature to a specified value.

$$
d_{e}=\left(\frac{\left(k_{e} t_{h}\right)^{2}}{2\left(\rho_{s} c_{p s} d_{s}\right)\left(\rho_{e} c_{p e}\right)\left(-\ln \left(1-\frac{T_{m}}{T_{h}}\right)\right)^{2}}\right)^{\frac{1}{3}}
$$

Substituting Equation (6) into Equation (4) produces an expression for mass per unit area as a function of structural thickness:

$$
m=\left(\frac{\left(\frac{\rho_{e} k_{e}}{\sqrt{c_{p e}}}\right)^{2} t_{h}^{2}}{2 \rho_{s} d_{s} c_{p s}\left(-\ln \left(1-\frac{T_{m}}{T_{h}}\right)\right)^{2}}\right)^{\frac{1}{3}}+\rho_{s} d_{s}
$$

The first term in Equation (7) represents the mass of insulator required to limit the structure to the specified maximum temperature and the second term represents structural mass. Equation (7) can be written as a function of $m_{s}$.

$$
m=\left(\frac{\left(\frac{\rho_{e} k_{e}}{\sqrt{c_{p e}}}\right)^{2} t_{h}^{2}}{2 c_{p s}\left(-\ln \left(1-\frac{T_{m}}{T_{h}}\right)\right)^{2}}\right)^{\frac{1}{3}} m_{s}^{-\frac{1}{3}}+m_{s}
$$

\section{IV.A. Material Properties and Insulator Mass}

The first term in Equation (8) represents the mass of insulator required to limit the structure to the specified maximum temperature. Inspection of this equation illustrates the effect of key parameters on 
required insulator mass.

$$
m_{e}=\left(\frac{\left(\frac{\rho_{e} k_{e}}{\sqrt{c_{p e}}}\right)^{2}}{c_{p s}\left(-\ln \left(1-\frac{T_{m}}{T_{h}}\right)\right)^{2}}\right)^{\frac{1}{3}}\left(\frac{t_{h}^{2}}{2 m_{s}}\right)^{\frac{1}{3}}
$$

From Equation (9) it is obvious that a longer heat pulse (increased $t_{h}$ ) will result in a larger required insulator mass. Similarly, a larger structural mass will provide more structural heat sink capacity and require less insulator mass. For a given structural mass and a particular transient heating pulse, required insulation mass will be determined by the thermal properties of the insulator and structure.

The specific heat capacities of insulators and structural materials vary significantly with temperature and the thermal Table 1. Simplified surface temperature histoconductivity of low density insulators is a function of both temperature and pressure. Therefore, it is not immediately obvious which material property values to use in the preceding equations to obtain results that are meaningful and useful. A method was previously devised ${ }^{1}$ to calculate an equivalent square heating pulse that approximates a surface temperature history on a vehicle during atmospheric entry, resulting in values of $t_{h}, T_{h}$ and $P_{a v g}$. These values are shown in Table 1 for the seven heating histories previously studied. ${ }^{1,2}$ The first four temperature histories were measured at four different locations ries

\begin{tabular}{c|ccrc}
\hline \hline Profile & $\begin{array}{c}t_{h} \\
\mathrm{~s}\end{array}$ & $\begin{array}{c}T_{h} \\
{ }^{\circ} \mathrm{C}\end{array}$ & $\begin{array}{c}P_{\text {avg }} \\
\mathrm{Pa}\end{array}$ & $\begin{array}{c}T_{k e} \\
K\end{array}$ \\
\hline BP9470 & 1273.6 & 838.6 & 98.0 & 792.0 \\
BP9678 & 1313.8 & 799.9 & 133.0 & 768.8 \\
BP9591 & 1206.6 & 804.0 & 99.7 & 771.3 \\
BP9489 & 1191.2 & 576.0 & 59.1 & 634.5 \\
ATSpA & 1914.4 & 871.2 & 1756.6 & 811.6 \\
RLVpA & 2042.1 & 861.8 & 1025.5 & 806.0 \\
RLV3c & 1919.6 & 727.0 & 1364.6 & 725.1 \\
\hline \hline
\end{tabular}
on the windward surface of a Shuttle Orbiter during a single atmospheric entry flight. ${ }^{3,4}$ It is interesting to note the variation in the equivalent square heating pulses that occur over just the windward surface in a single flight. The last three heating histories were predicted for a single point on the windward surface of three different proposed reusable launch vehicles. ${ }^{5,6}$ Also shown is the temperature ${ }^{1}$ at which the effective insulator thermal conductivity should be calculated.

$$
T_{k e}=T_{i}+0.6 T_{h} \text {, where } T_{i}=60^{\circ} \mathrm{F}, 289 \mathrm{~K}
$$

Each of the relevant thermal properties was examined independently ${ }^{1}$ to devise a method to calculate an equivalent constant value corresponding to a particular transient surface temperature history. The equivalent constant values for a particular surface heating history can be calculated as follows:

$$
\begin{gathered}
c_{p s}=c_{p s}\left(T_{c s}\right), \text { where } T_{c s}=T_{i}+\frac{T_{m}}{2}, \\
c_{p e}=c_{p e}\left(T_{c e}\right), \text { where } T_{c e}=T_{i}+T_{m}, \text { and } \\
k_{e}=k_{e}\left(T_{k e}, P_{a v g}\right) .
\end{gathered}
$$

Inspection of Equations (8) and (9) reveals that the thermal properties of the structure and insulator can each be grouped separately. These grouped properties have the potential to be useful for comparing the thermal effectiveness of both the structural and insulator materials.

The specific heat capacity and maximum allowable temperature of the structure directly affect the required insulation mass. However, the thermal properties of the structure are coupled to the amplitude of the equivalent square heating pulse, $T_{h}$. The following expression is a candidate figure of merit for the thermal effectiveness of the structural material:

$$
\beta_{s}=c_{p s}\left(-\ln \left(1-\frac{T_{m}}{T_{h}}\right)\right)^{2}
$$

A larger value of this parameter will lead to a smaller required mass of insulation. Therefore a structure with a high specific heat capacity and high maximum temperature limit will tend to require less insulation. 
For steady state heat conduction, minimizing the product of insulator density and thermal conductivity, $\rho_{e} k_{e}$, minimizes the mass of required insulator. ${ }^{6}$ However, a similar figure of merit for minimum mass of insulation subjected to transient heating has not been available. The grouping of insulator properties in Equation (9) suggests a candidate figure of merit for minimum mass insulation for a transient heating pulse (or at least for the simplified heat pulse in this derivation):

$$
\kappa_{e}=\frac{\rho_{e} k_{e}}{\sqrt{c_{p e}}}
$$

A smaller value of this parameter will lead to a smaller required mass of insulation. Therefore, a low mass insulator for the transient heat pulse should have a combination of low $\rho_{e} k_{e}$ and high $c_{p e}$.

\section{IV.B. Minimum Mass of Insulated Structure}

The mass of the insulated structure, calculated using Equation (8), is obviously a function of the mass of the structural material. Increasing the amount of structural mass linearly adds to the total mass, but results in a corresponding decrease in the mass of required insulation. Therefore, in the absence of any overriding structural requirements, there should be a structural mass for which the total mass is minimum. The structural mass for which the total mass is minimum can be readily calculated by taking the first derivative of Equation (8) with respect to $m_{s}$ and setting it equal to zero. The result can be solved for $m_{s}$ to produce:

$$
m_{s o}=54^{\left(-\frac{1}{4}\right)} \sqrt{t_{h}}\left(\frac{\left(\frac{\rho_{e} k_{e}}{\sqrt{c_{p e}}}\right)^{2}}{c_{p s}\left(-\ln \left(1-\frac{T_{m}}{T_{h}}\right)\right)^{2}}\right)^{\frac{1}{4}}
$$

Equation (16) is potentially useful for sizing insulated structure. The structure could first be sized to carry the design structural loads. The resulting equivalent structural mass can then be compared to that calculated using Equation (16). If the result of Equation (16) is greater, then increasing the structural mass to that value will result in the lowest combined mass of structure and insulator. However, if the result of Equation (16) is less, then adding additional structural mass will only increase the combined mass.

Substituting Equation (16) back into Equation (8) produces the following expression for the minimum mass of an insulated structure:

$$
\begin{gathered}
m_{\text {opt }}=(3+1) m_{\text {so }} \\
m_{\text {opt }}=2\left(\frac{2}{3}\right)^{\left(\frac{3}{4}\right)} \sqrt{t_{h}}\left(\frac{\left(\frac{\rho_{e} k_{e}}{\sqrt{c_{p e}}}\right)^{2}}{c_{p s}\left(-\ln \left(1-\frac{T_{m}}{T_{h}}\right)\right)^{2}}\right)^{\frac{1}{4}}
\end{gathered}
$$

The first term in parentheses in Equation 17 indicates the relative contributions of the insulation and structure, respectively, to the minimum total mass. For minimum total mass, Equation 17 predicts that the mass of the insulation will be three times the mass of the structure. Equation (18) defines the lower bound for the mass of an insulated structure subjected to a transient heat pulse. The magnitude of the minimum

total mass is solely a function of the duration of the equivalent square heating pulse, $t_{h}$, and the figures of merit defined in Equations (14) and (15).

\section{Analytical Predictions and Numerical Simulations}

Several numerical studies were performed to assess how the simple approximate solutions derived in this paper compare to more accurate numerical simulations of earth atmospheric entry heating on reusable launch vehicles. The same finite element model and heating histories ${ }^{1,2}$ were used for all of these numerical studies. The objectives of the numerical studies were: 1) to assess the accuracy of using Equations 6 and 9 to size insulation for a realistic transient heating pulse, 2) to determine the applicability of the figures of 
merit defined by Equations 14 and 15 to results of atmospheric entry simulations, and 3) to investigate the interplay between the mass of the structure and the corresponding mass of insulation required to limit the maximum structural temperature.

\section{V.A. Finite Element Model}

A one-dimensional finite element model was developed using the DOLFIN ${ }^{7}$ finite element library for the Python programming language. Using the DOLFIN library, linear one dimensional elements were used to discretize the spatial dimension and an implicit Crank-Nickelson time marching scheme was used to solve the weak formulation of the diffusion equation. The model consisted of a layer of insulator material in perfect contact with a layer of structural material similar to the configuration shown in Figure 1, except that the structure in the finite element model was not treated as a lumped heat capacitance. For the results shown in this paper, the model consisted of 50 elements through the thickness of the insulator and 2 elements through the thickness of the structure. The boundary condi-

Table 2. Thermal Properties of Four Structural Materials

\begin{tabular}{|c|c|c|c|}
\hline$T_{i}+T_{m}$ & $c_{p s}\left(T_{c s}\right)$ & $\beta_{s}(B P 7940)$ & $\beta_{s}(A T S p A)$ \\
\hline$K\left({ }^{\circ} \mathrm{F}\right)$ & $\frac{J}{k g K}\left(\frac{B t u}{l b m R}\right)$ & $\frac{J}{k g K}\left(\frac{B t u}{l b m R}\right)$ & $\frac{J}{k g K}\left(\frac{B t u}{l b m R}\right)$ \\
\hline \multicolumn{4}{|c|}{ Aluminum $2024 \rho_{s}=2803 \frac{\mathrm{kg}}{\mathrm{m}^{3}}\left(0.10 \frac{\mathrm{lbm}}{\mathrm{in}}\right)$} \\
\hline $422(300)$ & $900(0.215)$ & $27(0.006)$ & $25(0.006)$ \\
\hline $450(350)$ & $910(0.217)$ & $41(0.010)$ & $38(0.009)$ \\
\hline \multicolumn{4}{|c|}{ Graphite/epoxy $\rho_{s}=1576 \frac{\mathrm{kg}}{\mathrm{m}^{3}}\left(0.057 \frac{\mathrm{lbm}}{i n^{3}}\right)$} \\
\hline $366(200)$ & $913(0.218)$ & $9(0.002)$ & $8(0.002)$ \\
\hline $422(300)$ & $984(0.235)$ & $30(0.007)$ & $27(0.006)$ \\
\hline \multicolumn{4}{|c|}{ Beryllium Aluminum $\rho_{s}=2098 \frac{\mathrm{kg}}{\mathrm{m}^{3}}\left(0.076 \frac{\mathrm{lbm}}{\mathrm{in} \mathrm{n}^{3}}\right)$} \\
\hline $505(450)$ & $1690(0.404)$ & $151(0.036)$ & $138(0.033)$ \\
\hline $561(550)$ & $1735(0.415)$ & $267(0.064)$ & $244(0.058)$ \\
\hline \multicolumn{4}{|c|}{ Titanium 6Al-4V $\rho_{s}=4437 \frac{\mathrm{kg}}{\mathrm{m}^{3}}\left(0.16 \frac{\mathrm{lbm}}{\mathrm{in} \mathrm{n}^{3}}\right)$} \\
\hline $533(500)$ & $574(0.137)$ & $68(0.016)$ & $62(0.015)$ \\
\hline $700(800)$ & $594(0.142)$ & $270(0.064)$ & $242(0.058)$ \\
\hline
\end{tabular}

tion on the outer surface of the insulator consisted

of an imposed surface temperature that could be varied arbitrarily with time and updated at each time step of the solution. The inner surface of the structure was adiabatic. The material properties could be arbitrary functions of temperature and ambient pressure. Material property values for each finite element were updated at each time step of the analysis. Property values could be different for each element, but did not vary spatially within an element. Time steps between 1 and 5 seconds were used to calculate the results presented in this paper. The finite element simulation was iterated to determine the insulation thickness required to limit the structural temperature rise to the specified value. Each transient simulation was continued until the structural temperature had reached its maximum value.

\section{V.B. Thermal Properties of Structural Material}

Obviously, raising the structural temperature limit will decrease the amount of required insulation. Less commonly considered is the effect of the structural specific heat capacity on the required insulation. Equation 14 defines a candidate figure of merit that combines the effects of structural temperature limit and specific heat capacity on the amount of required insulation.

A numerical study was performed to assess how well the results of a series of full numerical simulations would correlate to the parameter $\beta_{s}$. To compare the effects of the structural thermal properties, it was necessary to fix all other parameters in the problem and vary only the choice of structural material. LI-900 tile was chosen as the insulator and the structural mass per unit area was fixed at $6.10 \frac{\mathrm{kg}}{\mathrm{m}^{2}}\left(1.25 \frac{\mathrm{lbm}}{\mathrm{ft}^{2}}\right)$. Two different

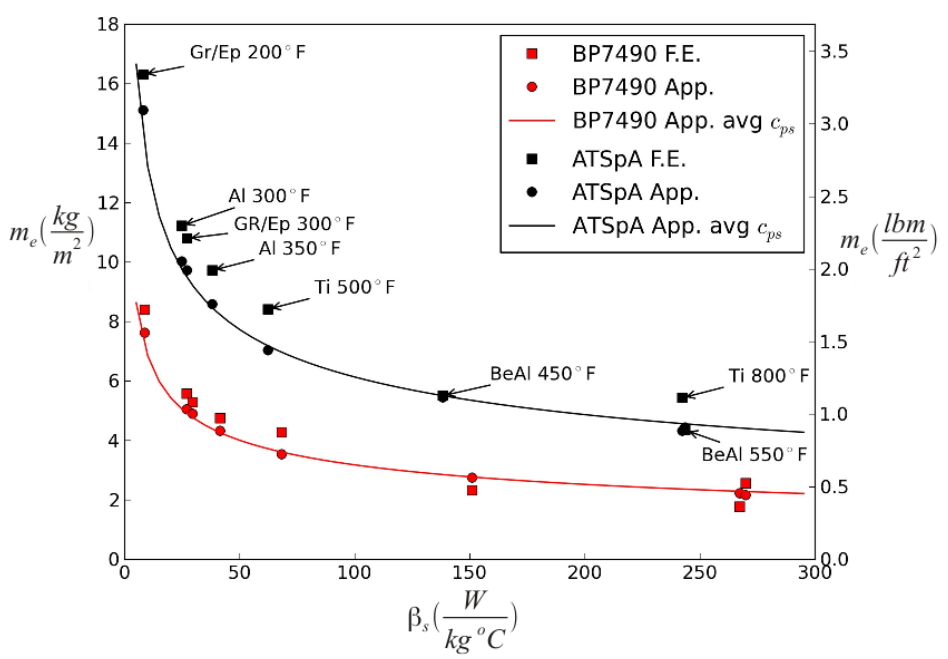

Figure 2. Effect of structural thermal properties on required insulator mass heating histories were considered, but results can only be directly compared for a particular heating history. Table 2 shows thermal properties for four structural materials chosen for the study. The temperature dependent properties for these materials are 
tabulated in another paper. ${ }^{1}$ The maximum allowable temperature for each material is application dependent, so an approximate upper and lower bound was chosen for each material. For each limiting temperature the structural specific heat capacity (Equation 11) and $\beta_{s}$ for each the two heating histories are shown in Table 2.

Iterative finite element analysis was used to size insulation for 16 cases ( 4 structural materials, 2 temperature limits, and 2 heating histories). The resulting insulation masses are shown in Figure 2 and indicated by the square symbols. For the ATSpA heating history, each particular structural material and temperature limit is identified in the figure. The square symbols are not labeled for the BP7490 heating history to avoid cluttering the figure. The approximate insulation masses, calculated using Equation 9, are indicated by circles corresponding to each finite element solution. For the Space Shuttle heating history, BP7490, half of the approximate solutions are within 10 percent of the finite element solutions and all of the approximate solutions are within 25 percent. For the reusable launch vehicle heating history, ATSpA, half of the approximate solutions are within 10 percent of the finite element solutions and all of the approximate solutions are within 20 percent. Because the value of $c_{p e}$ used in Equation 9 is a function of the maximum structural temperature limit (Equation 12), the approximate insulation masses are not solely a function of $\beta_{s}$. The solid lines in Figure 2 represent the approximate solution using a value of $c_{p e}$ that is an average of the 8 cases for each temperature history. The close agreement between the solid lines and circles indicates that, for LI-900 insulation, the variation of $c_{p e}$ with $T_{m}$ has little effect on the mass of required insulation. The solid lines clearly indicate the effect of $\beta_{s}$ on the required insulation mass predicted by the approximate solution. Remarkably, the finite element solutions closely follow this trend, even with four different structural materials. Therefore, the parameter $\beta_{s}$ appears to be a useful indicator of the thermal effectiveness of a structural material.

\section{V.C. Thermal Properties of Insulator}

To better understand the significance of the parameter defined in Equation 15 it is helpful to consider a range of candidate insulator materials that could be used for thermal protection on a reusable launch vehicle. A numerical study was performed to investigate the relative mass efficiency of a range of insulators for two different surface temperature histories (BP7490 and ATSpA) from Table 1. Five rigid ceramic tile materials (LI-900, LI-2200, FRCI12, AETB-8, AETB-16) and four flexible insulations (Saffil-3, Saffil-6, Qfiber-3, and Qfiber-6) were considered. The temperature and pressure dependent thermal properties of the three Space Shuttle Orbiter tile materials ((LI-900, LI-2200, and FRCI-12) were obtained from published tables. ${ }^{8}$ The thermal properties for AETB-8 and AETB-16 were calculated using the model developed by Daryabeigi ${ }^{9}$ and the flexible insulation properties were calculated using the models also developed by Daryabeigi. ${ }^{10}$ For this study, aluminum with a maximum temperature limit of $450 \mathrm{~K}\left(350^{\circ} \mathrm{F}\right)$ was chosen as the underlying structure. The structural mass per unit area was fixed at $6.10 \frac{\mathrm{kg}}{\mathrm{m}^{2}}\left(1.25 \frac{\mathrm{lbm}}{\mathrm{ft}}\right)$.

Table 3 shows the effective $c_{p e}$ (Equation 12) and $k_{e}$ (Equation 13) of each insulation for the two surface heating histories. The insulation figure of merit, calculated using Equation 15, is shown for each set

Table 3. Insulation figure of merit values for aerospace insulations

\begin{tabular}{|c|c|c|c|}
\hline Temp. History & $c_{p e}$ & & $\kappa_{e}=\frac{\rho_{e} k_{e}}{\sqrt{{ }^{c p e}}}$ \\
\hline & $\frac{W}{m K}$ & $\frac{J}{k g K}$ & $\frac{k g^{2}}{m^{3} s^{2} \sqrt{K}}$ \\
\hline \multicolumn{4}{|c|}{ LI-900 $\rho_{s}=144 \frac{\mathrm{kg}}{\mathrm{m}^{3}}\left(9 \frac{\mathrm{lbm}}{\mathrm{ft} \mathrm{t}^{3}}\right)$} \\
\hline BP 7490 & 949 & 0.03399 & 0.1589 \\
\hline ATSpA & 949 & 0.06060 & 0.2832 \\
\hline \multicolumn{4}{|c|}{ LI-2200 $\rho_{s}=352 \frac{\mathrm{kg}}{\mathrm{m}^{3}}\left(22 \frac{\mathrm{lbm}}{\mathrm{ft}^{3}}\right)$} \\
\hline BP7490 & 949 & 0.06046 & 0.6907 \\
\hline ATSpA & 949 & 0.08326 & 0.9512 \\
\hline \multicolumn{4}{|c|}{ FRCI-12 $\rho_{s}=192 \frac{\mathrm{kg}}{\mathrm{m}^{3}}\left(12 \frac{\mathrm{lbm}}{f t^{3}}\right)$} \\
\hline BP7490 & 949 & 0.04039 & 0.2517 \\
\hline ATSpA & 949 & 0.07212 & 0.4494 \\
\hline \multicolumn{4}{|c|}{ AETB-8 $\rho_{s}=128 \frac{\mathrm{kg}}{\mathrm{m}^{3}}\left(8 \frac{\mathrm{lbm}}{\mathrm{ft}^{3}}\right)$} \\
\hline BP7490 & 975 & 0.05702 & 0.2338 \\
\hline ATSpA & 975 & 0.08728 & 0.3578 \\
\hline \multicolumn{4}{|c|}{ AETB-16 $\rho_{s}=256 \frac{\mathrm{kg}}{\mathrm{m}^{3}}\left(16 \frac{\mathrm{lbm}}{\mathrm{ft}^{3}}\right)$} \\
\hline BP 7490 & 975 & 0.05927 & 0.4860 \\
\hline ATSpA & 975 & 0.08975 & 0.7360 \\
\hline \multicolumn{4}{|c|}{ Saffil-3 $\rho_{s}=48 \frac{\mathrm{kg}}{\mathrm{m}^{3}}\left(3 \frac{\mathrm{lbm}}{\mathrm{ft}}\right)$} \\
\hline BP7490 & 975 & 0.04352 & 0.0669 \\
\hline ATSpA & 975 & 0.07302 & 0.1123 \\
\hline \multicolumn{4}{|c|}{ Saffil-6 $\rho_{s}=96 \frac{\mathrm{kg}}{\mathrm{m}^{3}}\left(6 \frac{\mathrm{lbm}}{f t^{3}}\right)$} \\
\hline BP7490 & 975 & 0.02420 & 0.0744 \\
\hline ATSpA & 975 & 0.05489 & 0.1688 \\
\hline \multicolumn{4}{|c|}{ Qfiber-3 $\rho_{s}=48 \frac{\mathrm{kg}}{\mathrm{m}^{3}}\left(3 \frac{\mathrm{lbm}}{\mathrm{ft}^{3}}\right)$} \\
\hline BP7490 & 932 & 0.04106 & 0.0646 \\
\hline ATSpA & 932 & 0.07328 & 0.1152 \\
\hline \multicolumn{4}{|c|}{ Qfiber-6 $\rho_{s}=96 \frac{\mathrm{kg}}{\mathrm{m}^{3}}\left(6 \frac{\mathrm{lbm}}{\mathrm{ft}^{3}}\right)$} \\
\hline BP7490 & 932 & 0.02158 & 0.0679 \\
\hline ATSpA & 932 & 0.04975 & 0.1564 \\
\hline
\end{tabular}

of material properties. As shown in Table 3, the insulation effective material properties and figure of merit, $\kappa_{e}$, for a given insulation can be significantly different for different surface temperature histories. Therefore, insulations should be directly compared only for a given surface temperature history, structural material, and structural mass. 
The iterative finite element analysis, previously described, was used to calculate the required insulation mass for each insulation and surface heating history listed in Table 3. Figure 3 shows the required insulation masses versus the insulation figure of merit, $\kappa_{e}$. Each symbol shape represents the finite element solution for each of the insulations represented in Table 3 and the solid line represents the approximate solution calculated using Equation 9. The red line and symbols correspond to the BP7490 temperature history and the black line and symbols correspond to the ATSpA temperature history. For each temperature history, the finite element results for this wide range of insulators clearly follow the trend predicted by the approximate solution.

For both surface temperature histories the flexible insulation materials are more mass efficient than the rigid tile materials. For the Shuttle Orbiter surface temperature history (BP7490), the bulk of the heating takes place at such a low pressure that gas conduction is not significant and radiation dominates the heat transfer through the insulation. Therefore the density of the flexible insulation (affects the length of gas conduction path) has little effect on the thermal performance, so that all four flexible insulations have nearly the same mass efficiency. Conversely, the bulk of the heating for the reusable launch vehicle surface temperature history (ATSpA) occurs at a higher pressure at which gas conduction through the insulation is significant. Therefore, the lower density flexible insulations are more mass efficient than the higher density

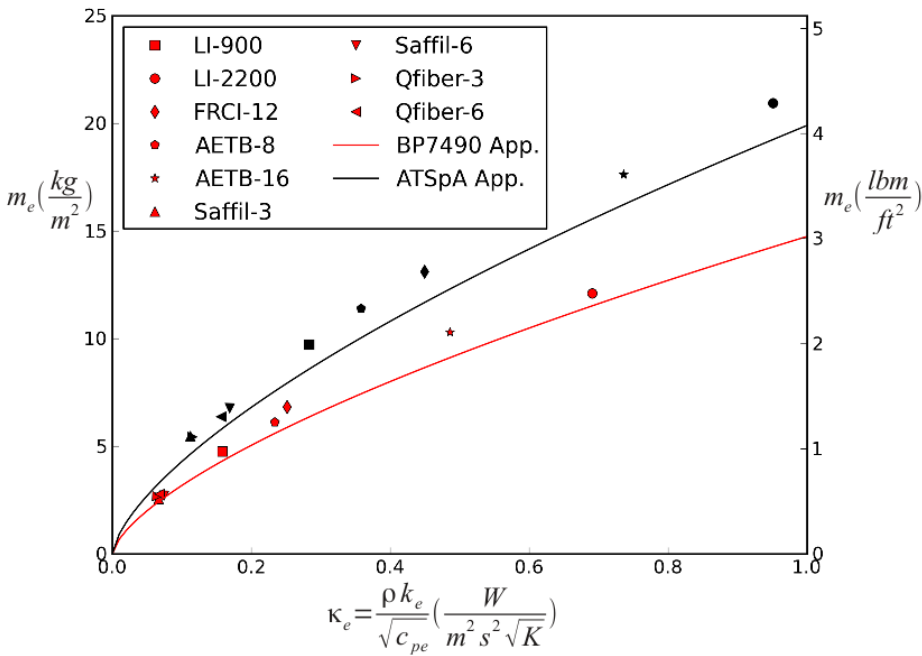

Figure 3. Required insulation mass for several insulators flexible insulations. For both surface temperature histories, lower density ceramic tiles tend to be more mass efficient than higher density tiles because heat transfer due to solid conduction becomes more significant with density. However, the denser LI-900 tile is more mass efficient than the AETB- 8 tile. The required insulation masses predicted by the approximate equation are within 15 percent of the finite element solutions.

\section{V.D. Interplay Between Mass of Insulator and Structure}

An additional numerical study was performed to gain a better understanding of the interplay between the mass of the structure and the mass of insulator required to limit the structural temperature. LI-900 ceramic tile was used as the insulator. Aluminum, with a maximum temperature limit of $450 \mathrm{~K}$ $\left(350^{\circ} \mathrm{F}\right)$, was chosen as the underlying structure. The structural mass per unit area was varied between 0.49 and $14.6 \frac{\mathrm{kg}}{\mathrm{m}^{2}}(0.1$ and 3.0 $\left.\frac{l b m}{f t^{2}}\right)$. For each of the two different surface temperature histories (BP7490 and ATSpA) from Table 1, iterative finite element analyses were performed for thirteen different values of structural mass per unit area to calculate the required insulation mass.

Results of this numerical study are shown in Figure 4. The red symbols and lines correspond to the BP7490 heating history and the black to the ATSpA heating history. The blue line is the mass of the structure, which was the same for both heating histories. Required insulation masses

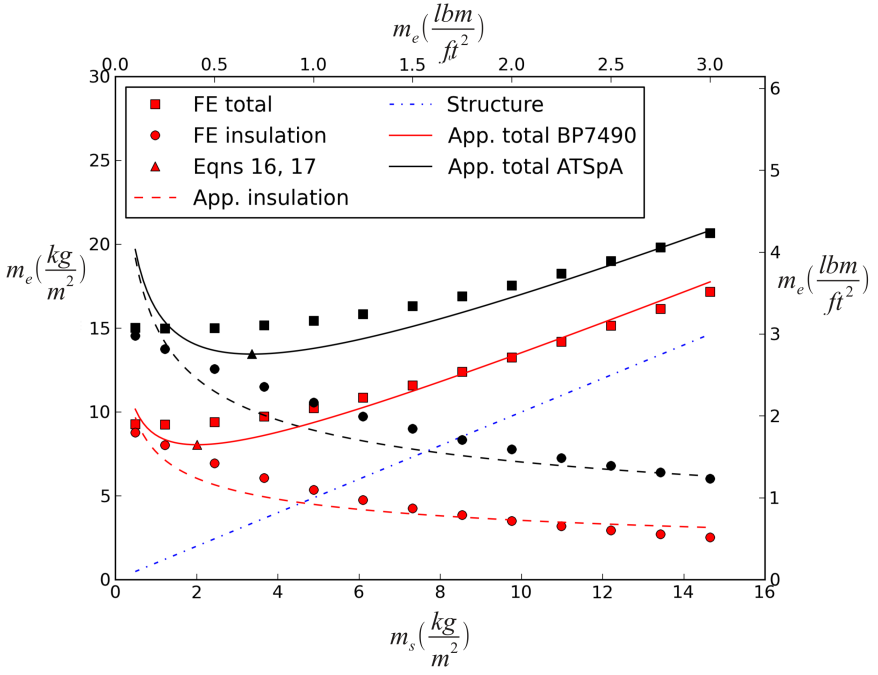

Figure 4. Mass of insulated structure as a function of structural mass 
calculated using the finite element analyses are represented by the solid circles. Dashed lines represent the corresponding approximate insulation masses calculated using Equation 9 with properties defined by Equations 11-13. The solid squares represent the sum of the finite element solutions for insulation mass and the structural mass. The solid lines represent the sum of the approximate solution for insulation mass and the structural mass. The solid triangles represent the minimum total mass calculated using Equations 16 and 18.

Comparison of the required insulation masses calculated using the finite element analysis and using Equation 9 (solid circles vs dashed lines in Figure 4) shows remarkably good agreement. Over the middle range of structural masses studied the approximate solution is within 15 percent of the finite element solution for both heating histories. However, for very low values of structural mass the approximate solution begins to sharply diverge from the finite element solution. Also, at the high end of the range of structural masses, the approximate solution for the BP7490 heating history is beginning to diverge from the finite element solution. This indicates that the approximate solution may become much less accurate when the ratio of insulation mass to structural mass becomes very high or very low. The total mass is obtained by simply adding the structural mass to the insulation mass, so the accuracy comparison between the finite element and approximate solutions is similar to that for the required insulation masses.

As the structural mass increases the required insulation mass decreases, so there is the potential for a combination of structure and insulator that produces the minimum total mass. For the finite element results the total mass minimum occurs at a structural mass value of about $1 \frac{\mathrm{kg}}{\mathrm{m}^{2}}\left(0.2 \frac{\mathrm{lbm}}{\mathrm{ft}}\right)$ for both heating histories. Equation 16 predicts a minimum at $2.1 \frac{\mathrm{kg}}{\mathrm{m}^{2}}$ for the BP7490 heating history and $3.6 \frac{\mathrm{kg}}{\mathrm{m}^{2}}$ for the ATSpA heating history. Although Equation 16 does not accurately predict the structural mass at which the total mass is a minimum, the values of the minimum total mass predicted using Equation 18 are within 10 percent of the finite element calculations for both heating histories. The three to one ratio of insulator mass to structural mass predicted to occur (Equation 17) at the minimum mass appears to be an artifact of error in the approximation.

The total mass curves in Figure 4 are relatively flat over a significant range of structural masses. Therefore, the mass (and thickness) of the insulator can be reduced by increasing the structural mass, for a relatively modest total mass penalty. Thinner vehicle walls, insulator plus structure, could improve vehicle packaging efficiency, perhaps resulting in a net mass savings.

\section{Summary}

Approximate analytical equations were developed for sizing the insulator thickness and mass required to maintain the insulated structural skin of hypersonic aerospace vehicles below a specified temperature. Manipulation of the equations revealed that the thermal properties of the insulator and of the structure could each be collected into a single term. These terms could be used as figures of merit to indicate the effect of the choice of insulator or structural material on the required insulator mass. An equation was also developed for the minimum total mass of an insulated structure sized to stay below the maximum structural temperature limit, ignoring other design considerations.

A one-dimensional, finite element, transient thermal analysis was used to perform numerical studies for comparison with the results of approximate equations developed in this paper. The finite element analysis included the effects of temperature and pressure dependent material properties. Time-accurate surface temperature histories and ambient pressure histories were also incorporated into the numerical models.

A numerical study was performed to compare the mass of insulation required to protect 4 different structural materials. Two different maximum structural temperatures were considered for each structural material. The insulation material and the structural mass were kept the same, but calculations were performed for two different surface temperature histories. The approximate solution predicted required insulation masses within 10 percent of the finite element results for more than half of the cases and within 25 percent for all of the cases considered. The finite element results exhibited a close correlation to the figure of merit, $\beta_{s}$, derived in this paper.

A second numerical study was performed to compare the mass efficiency of 9 different aerospace insulators, five ceramic tile materials and four flexible insulations. The structural material, mass and maximum temperature limit were held constant while the required insulation masses were calculated for the same two surface temperature histories. For the insulations considered, the required insulation masses predicted by the approximate equation were within 15 percent of the finite element solutions. The finite element results 
were closely correlated to the insulation efficiency figure of merit, $\kappa_{e}$, derived in this paper.

A third numerical study was performed to investigate the effect of structural mass on the mass of required insulation and to determine the minimum mass for an insulated structure designed with only thermal constraints. The structural material and maximum temperature, as well as the insulation material, were held constant and the required insulation masses were calculated for a range of structural masses and for the same two surface temperature histories. For very small or very large values of structural mass the approximate solution diverges from the finite element solutions, however, away from these extremes the approximate solution was within 15 percent of the finite element solution. The approximate equation for minimum total mass was within 10 percent of the finite element solution, however, the approximate equation predicted the minimum total mass at a much larger value of the structural mass than the finite element solution.

The approximate equations developed in this paper were shown to predict the results of much more complex finite element calculations with surprising accuracy for the wide range of cases considered in this paper. Achieving these accurate results requires carefully following the techniques developed for mapping the transient surface temperature histories to equivalent square temperature pulses and for calculating the effective property values to substitute into the approximate equations. These simple approximate equations have the potential to be very useful for the preliminary investigation of a wide range of design space to identify attractive regions for more detailed study. The figures of merit developed in this paper can also be helpful for choosing between available insulation and structural materials as well as providing guidance for developing more efficient materials.

\section{Acknowledgments}

The author would like to thank NASA Langley Research Center for the opportunity to perform this work under the Floyd Thompson Fellowship. Fellowship advisors, Professors Raphael Haftka and Bhavani Sankar from the University of Florida and Dr. William Garver from Lockheed Martin provided much helpful advice and encouragement. Dr. Garver initially indicated the strong need for the type of simple design tool developed in this paper. Colleagues, Dr. Kamran Daryabeigi and Carl Poteet provided valuable review and discussion of the work as it progressed.

\section{References}

${ }^{1}$ Blosser, M. L., "Analysis and Sizing for Transient Thermal Heating of Insulated Aerospace Vehicle Structures," to be published as NASA TP.

${ }^{2}$ Blosser, M. L., "An Analytical Solution for Transient Thermal Response of an Insulated Structure," 43rd AIAA Thermophysics Conference, New Orleans, LA (submitted for publication).

${ }^{3}$ Gibson, D. M. and et. al., "HYTHIRM Radiance Modeling and Image Analyses in Support of STS-119, STS-125 and STS-128 Space Shuttle Hypersonic Re-entries," AIAA Paper 2010-244, Jan. 2010.

${ }^{4}$ Zalameda, J. N. and et. al., "Application of a Near Infrared Imaging System for Thermographic Imaging of the Space Shuttle during Hypersonic Re-Entry," AIAA Paper 2010-245, Jan. 2010.

${ }^{5}$ Myers, D. E., Martin, C. J., and Blosser, M. L., "Parametric Weight Comparsion of Current and Proposed Thermal Protection System (TPS) Concepts," AIAA Paper 99-3459, June 1999.

${ }^{6}$ Blosser, M. L., Advanced Metallic Thermal Protection Systems for Reusable Launch Vehicles, Ph.D. dissertation, University of Virginia, Charlottesville, VA, May 2000.

${ }^{7}$ Logg, A. and Wells, G., "DOLFIN: Automated finite element computing," ACM Transactions on Mathematical Software, Vol. 37, No. 2, 2010, pp. 28.

${ }^{8}$ Williams, S. D. and Curry, D. M., "Thermal Protection Materials: Thermophysical Property Data," NASA RP-1289, 1992.

${ }^{9}$ Daryabeigi, K., Cunnington, G. R., and Knutson, J. R., "Heat Transfer Modeling for Rigid High-Temperature Fibrous Insulation," 43rd AIAA Thermophysics Conference, New Orleans, LA (submitted for publication).

${ }^{10}$ Daryabeigi, K., Cunnington, G. R., Miller, S. D., and Knutson, J. R., "Combined Heat Transfer in High-Porosity HighTemperature Fibrous Insulations: Theory and Experimental Validation," AIAA Paper 2010-4660, June 2010. 